\title{
The Effects of a Simulated Workday of Prolonged Sitting on Seated versus Supine Blood Pressure and Pulse Wave Velocity in Adults with Overweight/Obesity and Elevated Blood Pressure
}

\author{
Abdullah Bandar Alansare ${ }^{a, b}$ Robert J. Kowalsky ${ }^{c}$ Melissa A. Jones ${ }^{a}$ \\ Sophy J. Perdomo ${ }^{a}$ Lee Stoner ${ }^{d}$ Bethany Barone Gibbs ${ }^{\text {a }}$ \\ aDepartment of Health and Physical Activity, College of Education, University of Pittsburgh, Pittsburgh, PA, USA; \\ ${ }^{b}$ Department of Exercise Physiology, College of Sport Sciences and Physical Activity, King Saud University, Riyadh, \\ Saudi Arabia; ' Department of Health and Kinesiology, College of Education and Human Performance, Texas A\&M \\ University-Kingsville, Kingsville, TX, USA; ${ }^{\mathrm{D} D e p a r t m e n t}$ of Sport and Exercise, University of North Carolina, \\ Chapel Hill, NC, USA
}

\section{Keywords}

Pulse wave velocity $\cdot$ Blood pressure $\cdot$ Heart rate $\cdot$ Prolonged sitting $\cdot$ Posture $\cdot$ Desk work

\begin{abstract}
We evaluated the effects of a simulated workday of prolonged sitting on blood pressure (BP) and pulse wave velocity (PWV) and examined whether posture (seated vs. supine) affected responses. Participants $(n=25)$ were adults, with overweight/obesity and elevated BP, and performed seated desk work for $7.5 \mathrm{~h}$. BP and PWV were measured in seated and supine postures at baseline (7:15 a.m.), midday (12:05 p.m.), and afternoon (4:45 p.m.). Generalized linear mixed models evaluated the effects of prolonged sitting on BP and PWV within each posture and interactions by posture and sex. In the recommended postures, seated BP and supine carotid-femoral pulse wave velocity (cfPWV) and carotid-ankle pulse wave velocity (caPWV), but not carotid-radial pulse wave velocity (crPWV), significantly increased over the simulated seated workday (all $p<0.05$; effect sizes [d] ranged from 0.25 to 0.44$)$. Whilst no posture-by-time interactions were observed $(p>0.05), \mathrm{BP}, \mathrm{caPWV}$, and crPWV were higher when seated versus supine (main effects of posture $p<0.05$;
\end{abstract}

$d$ ranged from 0.30 to 1.04). Exploratory analysis revealed that females had greater seated BP responses ( $p$ for interaction <0.05); seated PWV and supine BP and PWV responses were similar by sex ( $p$ for interaction $>0.05$ ). A simulated workday of prolonged sitting increased seated BP and supine cfPWV and caPWV, and posture minimally influenced these responses. These results add to the evidence suggesting a deleterious effect of prolonged sitting on cardiovascular health.

(c) 2020 S. Karger AG, Basel

\section{Introduction}

Sedentary behavior is any waking activity that has an energy cost of $\leq 1.5$ metabolic equivalents and occurs in a lying, reclining, or seated posture [1]. High levels of prolonged sedentary behaviors, such as prolonged sitting, have been associated with cardiovascular disease and allcause mortality in longitudinal studies [2, 3]. In the United States, prolonged sitting is especially prevalent among individuals employed in sedentary occupations, who are sedentary for approximately $11 \mathrm{~h}$ per day [4]. Thus, the attention of public health organizations and need for in-

$\begin{aligned} & \text { karger@karger.com } \\ & \text { www.karger.com/jvr }\end{aligned}$
Karger ${ }^{\prime /}$

Abdullah Bandar Alansare

Department of Health and Physical Activity, University of Pittsburgh 32 Oak Hill Court

Pittsburgh, PA 15261 (USA)

aba79@pitt.edu 
creased research on the cardiovascular risks of prolonged sitting has recently grown [2-6].

Several studies have evaluated acute cardiovascular responses, such as blood pressure (BP), across various durations (i.e., 2, 3, 7, and $8 \mathrm{~h}$ ) of prolonged sitting [7-12]. Recently, a handful of studies have also measured the effects of prolonged sitting on pulse wave velocity (PWV) $[8,10,13]$, a subclinical vascular measure where acute increases likely reflect adverse changes in vascular function such as increases in smooth muscle tone [7-10, 14, 15]. Yet, findings from these studies are not consistent in that some, but not all, report that BP, PWV, or both increase following prolonged sitting [7-10, 12, 14]. Several factors such as different durations of prolonged sitting, postural positioning when measuring $\mathrm{BP}$ and $\mathrm{PWV}$, allowance of restroom breaks during prolonged sitting, and different sample characteristics might have contributed to these inconsistent findings $[16,17]$. Studies that consider these methodological factors are needed to better understand the effects of prolonged sitting on BP and PWV. Such studies could shed light on the mechanisms through which prolonged sitting impacts cardiovascular health. Further, acute studies of longer durations of prolonged sitting (i.e., 7-8 h) that simulate actual working conditions are especially relevant to guide public health recommendations for prolonged sitting in desk workers.

Postural positioning during $\mathrm{BP}$ and $\mathrm{PWV}$ measurement is an important protocol design consideration that could impact responses to prolonged sitting. The cardiovascular system reacts differently in various postures, such as lying, sitting, standing, or transitioning, due to muscular pump, blood flow, gravity, and autonomic responses [18-22]. As a consequence, BP and PWV differ significantly when posture is altered (i.e., supine, head-up tilt, head-down tilt, or Fowler's position) [23]. Importantly, some cardiovascular measurements, especially PWV, are currently recommended to be measured in a supine posture [24]. Yet, performing such supine measurements requires an interruption of the prolonged sitting bout and could also compromise accuracy. Researchers have recently begun to consider postural positioning when conducting cardiovascular measurements during prolonged sitting protocols, such as using passive participant transfer from seated to supine postures for assessments [8]. Furthermore, a recent study has revealed that some cardiovascular functions, specifically systolic BP (SBP), appear to take longer than recommended time ( $25 \mathrm{vs.} 5 \mathrm{~min}$ ) to reach the steady state $[25,26]$ and allow for accurate measurements. This, in turn, could potentially affect the accuracy of and lead to greater variance in seated versus supine vascular measurements including BP and PWV. Yet, no studies have directly compared cardiovascular responses to prolonged sitting when assessed in a seated versus supine posture, leaving the effect of measurement posture unclear.

Therefore, this study aimed to (1) examine BP and PWV responses across a simulated workday of prolonged sitting according to currently recommended (seated BP and supine PWV) and alternate postures (supine BP and seated PWV) $[24,26]$ and (2) investigate whether posture (seated vs. supine posture) affects the $\mathrm{BP}$ and $\mathrm{PWV}$ responses to prolonged sitting. It was hypothesized that seated BP and supine PWV would significantly increase across the simulated workday of prolonged sitting. It was also hypothesized that seated BP and PWV responses would be significantly greater than supine BP and PWV following the simulated workday of prolonged sitting.

\section{Materials and Methods}

This study is a secondary analysis of data from a randomized crossover trial, described in detail elsewhere [6]. In brief, the study compared cardiovascular responses across 2 simulated workdays, prolonged sitting versus alternating standing and sitting using a sit-stand desk. The current study only uses data collected during the prolonged sitting experimental condition.

\section{Participants}

As previously described, 25 working-aged adults were enrolled in the study. The inclusion criteria in this study were (a) 20-65 years old, (b) SBP between 120 and $159 \mathrm{~mm} \mathrm{Hg}$ or diastolic BP (DBP) between 80 and $99 \mathrm{~mm} \mathrm{Hg}$, which were classified as prehypertension or stage I hypertension according to the JNC7 guidelines at the time when the study was conducted [27], and (c) overweight or stage I/II obesity with a BMI of 25.0 to $<40.0 \mathrm{~kg} / \mathrm{m}^{2}$. Participants were excluded for the following: (a) SBP $\geq 160 \mathrm{~mm} \mathrm{Hg}$ or $\mathrm{DBP} \geq 100 \mathrm{~mm} \mathrm{Hg}$, (b) atrial fibrillation or a cardiovascular event in the past 6 months, (c) using antihypertensive, glucoselowering, or any other drugs known to influence cardiovascular responses, (d) current treatment for cancer or other serious medical condition, (e) current smoker, (f) pregnant in the past 6 months or breastfeeding in the past 3 months, ( $g$ ) performing moderateto-vigorous exercise $\geq 90$ min per week over the past 3 months, or (h) currently enrolled in a weight loss program. The study was approved by the Institutional Review Board at the University of Pittsburgh. All participants provided informed consent prior to participating in any research procedures.

\section{Experimental Procedures}

Participants took part in a simulated workday of prolonged sitting. The experimental visit lasted approximately $10 \mathrm{~h}$ and included morning (7:15 a.m.), midday (12:05 p.m.), and afternoon (4:45 p.m.) cardiovascular testing, standard breakfast and lunch meals, and morning and afternoon sessions of prolonged sitting ( $3 \mathrm{~h}$ and 
40 min each) (shown in Fig. 1). During the seated periods, participants sat continuously at a standard desk with feet on the floor and performed office work, with the exception of necessary restroom breaks (mean $=3.7 \pm 1.3$ breaks).

Participants reported to our laboratory between 07:00 and 07:30 a.m. Upon arriving, adherence to previsit instructions including abstention from food, nicotine, and alcohol for $12 \mathrm{~h}$ and abstention from exercise and caffeine for $24 \mathrm{~h}$ was verbally confirmed. Next, following a 10-min rest in a seated posture, morning BP and PWV measurements were completed. Next, participants rested in a supine posture for $10 \mathrm{~min}$, and then cardiovascular measurements were repeated. Though we considered counterbalancing the order of BP and PWV measurements across the seated and supine postures, a systematic order (seated then supine) was purposefully selected. This choice allowed us to capture the effects of prolonged sitting in the seated posture during the protocol, avoiding any potential effects due to postural interruption. Thereafter, a standardized breakfast was distributed to participants followed by a restroom break if needed. The standardized meals (breakfast and lunch) were designed to each fulfill $30 \%$ of participants' daily caloric need during low activity level [28]. These meals comprised approximately 55$60 \%$ carbohydrate, $10-15 \%$ protein, and $25-30 \%$ fat.

Then, participants begun their morning prolonged sitting period ( $3 \mathrm{~h}$ and $40 \mathrm{~min}$ ) at a standard desk equipped with a phone and a computer with internet connection. During the seated period, participants were required to complete personal, job-related, and desk-based work, with no specific restrictions. Participants were also allowed to use their personal phone or the restroom as needed. During the final $30 \mathrm{~min}$ (beginning at $3 \mathrm{~h}$ and $10 \mathrm{~min}$ into the seated work period), participants were required to stay seated to limit influence of movement on cardiovascular measurements at the end of the work period.

Following the morning working session, midday measurements of BP and PWV were completed with the same protocol as the morning measurements. Then, participants consumed a standardized lunch followed by a restroom break if needed. Thereafter, participants completed the afternoon prolonged sitting session ( $3 \mathrm{~h}$ and $40 \mathrm{~min}$ ) followed by BP and PWV measurements with the same protocol as the morning period.

\section{Measurements}

Participants' demographic characteristics were self-reported on standard questionnaires. Body height was measured in duplicate by using a wall-mounted stadiometer (Perspective Enterprises, Portage, MI, USA), whereas body weight was measured in duplicate by using a digital scale (WB-110A; Tanita, Japan). Duplicate measures were averaged.

\section{Blood Pressure}

BP was measured by using the HEM-705 oscillometric device (Omron Healthcare, Inc., Lake Forest, IL, USA) which has previously been validated $[29,30]$. During eligibility screening, arm circumference was measured by using a Gulick tape measure to determine the proper cuff size for each participant. Prior to BP measurement, participants sat in a clinical chair for at least $10 \mathrm{~min}$ with back supported, feet on the floor, and arms supported at heart level [26]. Then, BP was measured in both arms; a second measure of $\mathrm{BP}$ was taken in the arm that had the higher initial pressure, with 1-min intervals between all measurements. The average of the 2 measurements was used for eligibility. Thereafter, the same arm
Morning assessment (7:15 a.m.):

Seated assessments: 10-min rest, BP, PWV

Transition to supine

Supine assessments: 10-min rest, BP, PWV

Breakfast

Morning seated work session (8:25 a.m.):

Participants were allowed to:

- Complete personal job-related tasks in a seated posture

- Take restroom breaks ad libitum, except during the $30 \mathrm{~min}$ prior to assessments

Mid-day assessment (12:05 p.m.):

Seated assessments: HR, BP, PWV

Transition to supine

Supine assessments: $10-$ min rest, BP, PWV

Lunch

Afternoon seated work session (1:05 p.m.):

Participants were allowed to:

- Complete personal job-related tasks in a seated posture

- Take restroom breaks ad libitum, except during the 30 min prior to assessments

Afternoon assessment (4:45 p.m.):

Seated assessments: HR, BP, PWV

Transition to supine

Supine assessments: 10-min rest, BP, PWV

Fig. 1. Experimental protocol. All participants reported to the laboratory between 7:00 and 7:30 a.m. BP and PWV, respectively, were measured 3 times (morning, midday, and afternoon) in a seated posture followed by supine. Standardized meals were consumed immediately after morning and afternoon assessments. Then, participants began their morning and afternoon prolonged sitting sessions $(3 \mathrm{~h}$ and 40 min per session) during which they performed self-provided desk work and were allowed to take necessary restroom breaks. BP, blood pressure; PWV, pulse wave velocity; HR, heart rate.

and cuff size were used for measurement during experimental visits. During experimental morning, midday, and afternoon assessments, BP was measured twice each in a seated posture and in a supine posture. The measurements were completed after at least $10 \mathrm{~min}$ of assuming each posture. The average of both measurements was used in the analysis. Mean arterial pressure (MAP) was calculated using the following formula: $\mathrm{MAP}=\mathrm{DBP}+1 / 3$ [SBP - DBP]. For descriptive purposes, heart rate (HR) was measured concurrently by using the oscillometric BP monitor and averaged across repeated measures for analysis.

\section{Pulse Wave Velocity}

Following the current guideline [24], carotid-ankle PWV (caPWV), carotid-radial PWV (crPWV), and carotid-femoral PWV (cfPWV) were measured using tonometry via Complior Analyse (ALAM Medical, Vincennes, France). Arterial path lengths were measured as follows: cfPWV was estimated as the sternal notch- 
Table 1. Participant characteristics

\begin{tabular}{lcccc}
\hline & Overall $(n=25)$ & Male $(n=16)$ & Female $(n=9)$ & $p$ value \\
\hline Age, years & $42(12)$ & $40(12)$ & $47(11)$ & 0.120 \\
Occupational status & & & & \\
$\quad$ Full-time & $17(68.0 \%)$ & $11(68.8 \%)$ & $6(66.7 \%)$ & 0.976 \\
$\quad$ Part-time & $5(20.0 \%)$ & $3(19.8 \%)$ & $2(22.2 \%)$ & 0.946 \\
$\quad$ Student & $3(12.0 \%)$ & $2(12.5 \%)$ & $1(11.1 \%)$ & 0.785 \\
SBP, mm Hg & $128(14)$ & $128(12)$ & $82(17)$ & 0.831 \\
DBP, mm Hg & $81(10)$ & $81(8)$ & $96(14)$ & 0.427 \\
MAP, mm Hg & $96(11)$ & $70(10)$ & $74(14)$ & 0.580 \\
HR, beats/min & $71(11)$ & $32.3(5.3)$ & $31.1(4.8)$ & \\
BMI, kg/m & $31.9(5.0)$ & &
\end{tabular}

Data are presented as mean (SD) or $n(\%)$. $p$ value represents between-sex comparison. SBP, systolic blood pressure; DBP, diastolic blood pressure; MAP, mean arterial pressure; HR, heart rate.

to-femoral artery distance minus the sternal notch-to-carotid artery distance; crPWV was measured as the direct distance between the carotid and radial arteries; caPWV was measured as the distance between the carotid and posterior tibial arteries. Sensors were simultaneously placed at the carotid, radial, femoral (supine only), and posterior tibialis arterial sites. Three high-quality PWV scans ( 10 waveforms each) were obtained for each site. PWV was calculated for each scan as the difference in path length divided by the difference in time for the pulse wave to reach each site; calculated PWVs for each site were averaged. The acceptable coefficient of variation within scan was set as $<10 \%$ across waveforms. Scans that resulted in coefficients of variation over $10 \%$ were rejected and were not averaged. Following $10 \mathrm{~min}$ in a seated posture, caPWV and crPWV were measured from each participant. Of note, the femoral artery was not accessible when seated, and, thus, cfPWV was not measured in the seated posture. Next, participants transitioned themselves several feet to a supine posture on an exam table, rested for $10 \mathrm{~min}$, and then were measured for caPWV, crPWV, and cfPWV. A recent study from our laboratory examining the properties of crPWV and caPWV in seated and supine postures found these measures had acceptable-to-good reliability (ICC ranging from 0.636 to 0.759 ) but poor-to-good validity ( $r$ ranging from 0.227 to 0.812 ) for crPWV and caPWV across postures [31].

\section{Statistics}

The sample size for the parent study $(n=25)$ was predetermined to detect a difference in repeated BP measures between experimental conditions (sit-stand vs. prolonged sitting) with the following assumptions: an effect size of 0.25 , assuming a withinsubject correlation of $0.7, \alpha=0.05$, and $80 \%$ power [6]. The characteristics of the participants, overall and by sex, were summarized as means and standard deviations (SD) unless otherwise indicated. In addition, independent $t$ tests or $\chi^{2}$ tests examined sex difference in participant characteristics. To examine the first hypothesis of whether prolonged workday sitting affected cardiovascular responses, generalized linear mixed (GLM) models initially evaluated the effects of time separately on seated and supine BP and $\mathrm{PWV}$ in response to the simulated workday of prolonged sitting. The $\beta$ coefficients for time from these models represent the response across the entire experimental visit, including baseline, midday, and afternoon assessments. Because a recent study has revealed sex differences in cardiovascular responses to prolonged sitting [10], we repeated analyses after stratification by sex. For the second hypothesis of whether cardiovascular responses differed by posture, GLM models were expanded to evaluate the effects of time (average change across the entire experimental protocol), posture (sit vs. supine), and their interaction on BP and PWV in response to the simulated workday of prolonged sitting. Additional exploratory testing evaluated three-way interactions by sex. Lastly, as BP and HR can influence PWV, we repeated analyses of PWV responses with covariate adjustment for HR and SBP. The significance level was set as $\alpha<0.05$. Stata version 14 (StataCorp, LLC, College Station, TX, USA) was used for all data analysis.

\section{Results}

All participants completed the experimental session with BP, supine cfPWV, and seated and supine crPWV assessments $(n=25)$. Four caPWV scans did not meet quality standards, resulting in a sample size of $n=21$ for this outcome. More than half of the participants reported male sex, with average BP in the prehypertensive range and average BMI classified as obese. Characteristics were similar in male and female participants (Table 1).

$B P$ and PWV Responses across the Simulated Workday

Table 2 shows BP and PWV responses to the simulated workday of prolonged sitting across the entire experimental visit by measurement posture. For the currently recommended BP measurement posture, seated SBP, DBP, and MAP significantly increased over time with small-to-moderate effect sizes. Though not currently recommended, supine BP was also examined and reported in Table 2. Only supine SBP significantly increased over 
Table 2. BP, HR, and PWV responses across a simulated workday by posture (effect of time)

\begin{tabular}{|c|c|c|c|c|}
\hline & Posture & $\beta(\mathrm{SE})$ & $p$ value & $d$ \\
\hline \multirow[t]{2}{*}{ SBP (mm Hg) } & Supine & $3.36(1.63)$ & 0.039 & 0.28 \\
\hline & Seated $^{\mathrm{a}}$ & $5.84(1.79)$ & 0.001 & 0.43 \\
\hline \multirow[t]{2}{*}{ DBP (mm Hg) } & Supine & $1.36(1.41)$ & 0.334 & 0.13 \\
\hline & Seated $^{\mathrm{a}}$ & $3.20(1.31)$ & 0.015 & 0.30 \\
\hline \multirow[t]{2}{*}{ MAP (mm Hg) } & Supine & $1.96(1.30)$ & 0.131 & 0.18 \\
\hline & Seated $^{\mathrm{a}}$ & $4.24(1.26)$ & 0.001 & 0.40 \\
\hline \multirow[t]{2}{*}{ HR (beats/min) } & Supine & $-0.79(1.08)$ & 0.462 & 0.07 \\
\hline & Seated & $-2.58(1.57)$ & 0.099 & 0.23 \\
\hline \multirow[t]{2}{*}{$\operatorname{crPWV}(\mathrm{m} / \mathrm{s})$} & Supine ${ }^{a}$ & $0.25(0.23)$ & 0.273 & 0.16 \\
\hline & Seated & $0.20(0.02)$ & 0.314 & 0.15 \\
\hline \multirow[t]{2}{*}{$\mathrm{caPWV}(\mathrm{m} / \mathrm{s})$} & Supine $^{\mathrm{a}}$ & $0.45(0.18)$ & 0.014 & 0.25 \\
\hline & Seated & $0.39(0.40)$ & 0.331 & 0.25 \\
\hline $\operatorname{cfPWV}(\mathrm{m} / \mathrm{s})$ & Supine ${ }^{\mathrm{a}}$ & $0.52(0.20)$ & 0.010 & 0.44 \\
\hline
\end{tabular}

SE, standard error; $d$, Cohen's $d$; SBP, systolic blood pressure; DBP, diastolic blood pressure; MAP, mean arterial pressure; HR, heart rate; crPWV, carotid-radial pulse wave velocity; caPWV, carotid-ankle pulse wave ve-

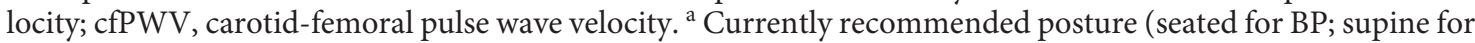
PWV). Bold indicates significant change $(p<0.05)$.

time with a small-to-moderate effect size, while DBP and MAP did not. In addition, seated and supine HR did not significantly change over time with both having small effect sizes. The exploratory analysis by sex revealed several significant sex-by-time interactions with prolonged sitting. Females had greater responses than males in seated DBP and MAP with prolonged sitting (interaction $p<$ $0.05)$; the effects of prolonged sitting on seated SBP and all supine BP were similar in females and males (online suppl. Table 1; for all online suppl. material, see www. karger.com/doi/10.1159/000510294).

For the currently recommended PWV measurement posture, Table 2 shows that supine cfPWV and caPWV significantly increased over time with small-to-moderate effect sizes. However, supine crPWV did not significantly change over time with small effect size. Though not currently recommended, seated PWV was also measured for caPWV and crPWV. Neither seated caPWV nor crPWV significantly changed over time, with both having small effect sizes. The exploratory analysis by sex revealed no significant sex-by-time interactions with prolonged sitting. However, increases in supine cfPWV, caPWV, and crPWV were more apparent in males (all $p<0.05$ ) versus females (all $p>0.05$ ) (online suppl. Table 1).

\section{The Influence of Posture on Responses across the \\ Simulated Workday}

Trajectories of BP and HR across the simulated workday by posture are shown in Figure 2. Differences in the effects of time across measurement postures were small and nonsignificant for SBP, DBP, MAP, and HR (interaction $\beta$, all $p>0.05$, reported in Fig. 2). However, there were moderate posture effects where SBP, DBP, MAP, and $\mathrm{HR}$ were significantly higher when seated compared to supine (all $p<0.05$, shown in Fig. 2). When combined across postures, there were overall small-to-moderate significant time effects of prolonged sitting for SBP and MAP but not significant for DBP or HR (shown in Fig. 2).

Trajectories of cfPWV, crPWV, and caPWV across the simulated workday by posture are shown in Figure 3. Because cfPWV was not measured in a seated posture, only the supine trajectory can be reported and was found to increase with a moderate effect size across the simulated workday (shown in Fig. 3). The difference in the effect of time across measurement postures was again trivial for crPWV and caPWV (interaction $\beta$ had $p>0.05$ ). There were small and large posture main effects, respectively, for crPWV $(d=0.30)$ and caPWV $(d=1.13)$, where PWVs were significantly higher in the seated compared to the supine posture. In contrast, considering seated and supine together, crPWV and caPWV did not change significantly over time across the simulated seated workday with both having small effect sizes (shown in Fig. 3).

Due to the potential influence of sex on cardiovascular responses to prolonged sitting, analyses were repeated with the addition of sex interaction terms; all were not significant $(p>0.05$; shown in online suppl. Fig. 1,2$)$. Furthermore, the analyses of PWV responses were re- 


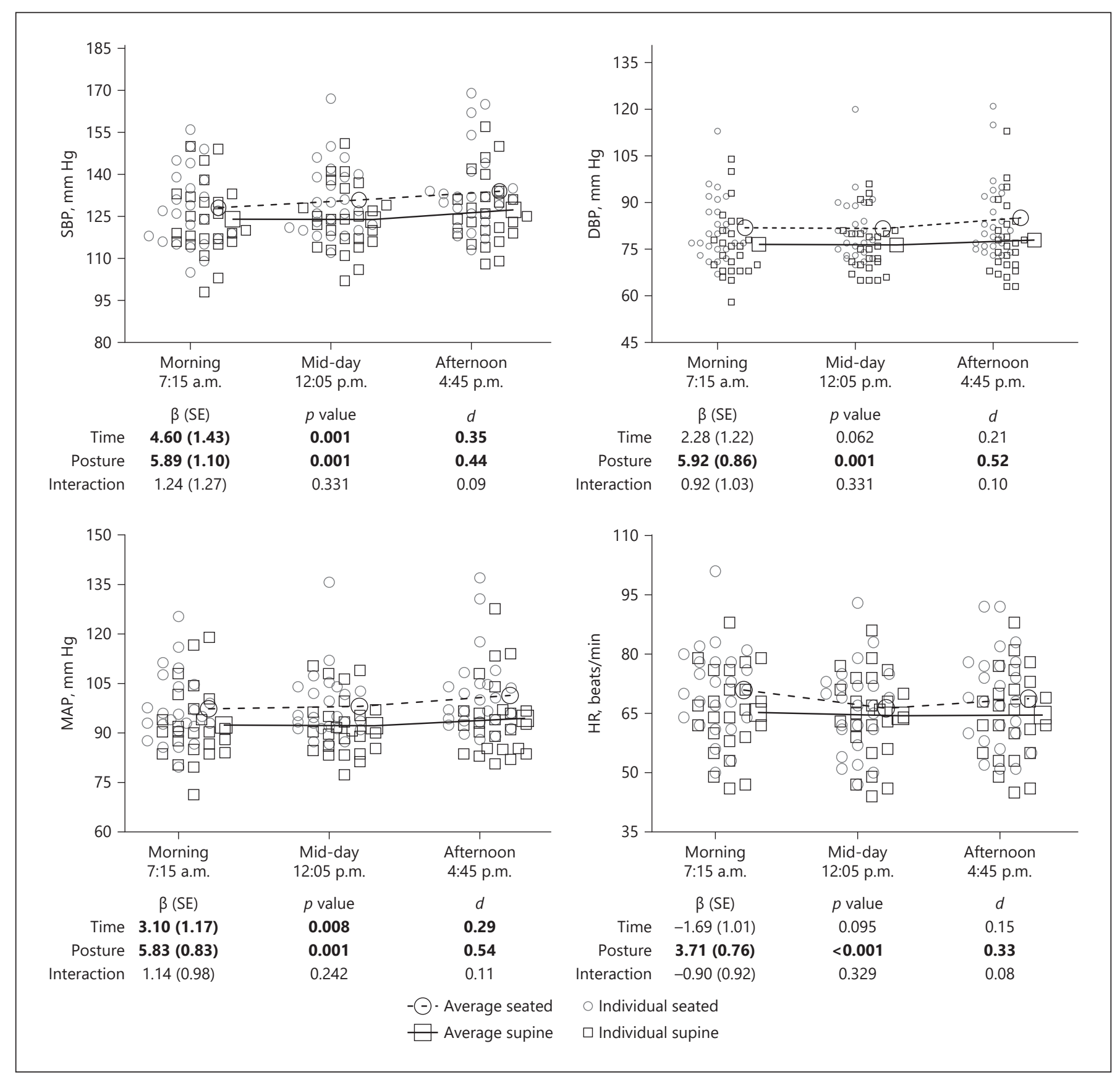

Fig. 2. $B P$ and $H R$ responses to prolonged sitting in a seated versus supine posture. GLM models examined the main effects of time, posture, and their interaction on BP and HR responses. $\beta$ coefficients represent change in $\mathrm{BP}$ or $\mathrm{HR}$ over the entire experiment (main effect of time), differences in BP or HR comparing sitting to

peated adjusting for SBP and HR. The adjusted models revealed similar PWV responses to prolonged sitting and across measurement postures (shown in online suppl. Table 2). supine (main effect of posture), and their interaction. $d$, Cohen's $d$; DBP, diastolic blood pressure; GLM, generalized linear mixed; $\mathrm{HR}$, heart rate; MAP, mean arterial pressure; SBP, systolic blood pressure; SE, standard error. Bold indicates significant effect $(p<$ $0.05)$.

\section{Discussion}

The primary findings of this study were that a simulated workday of prolonged sitting resulted in increases 

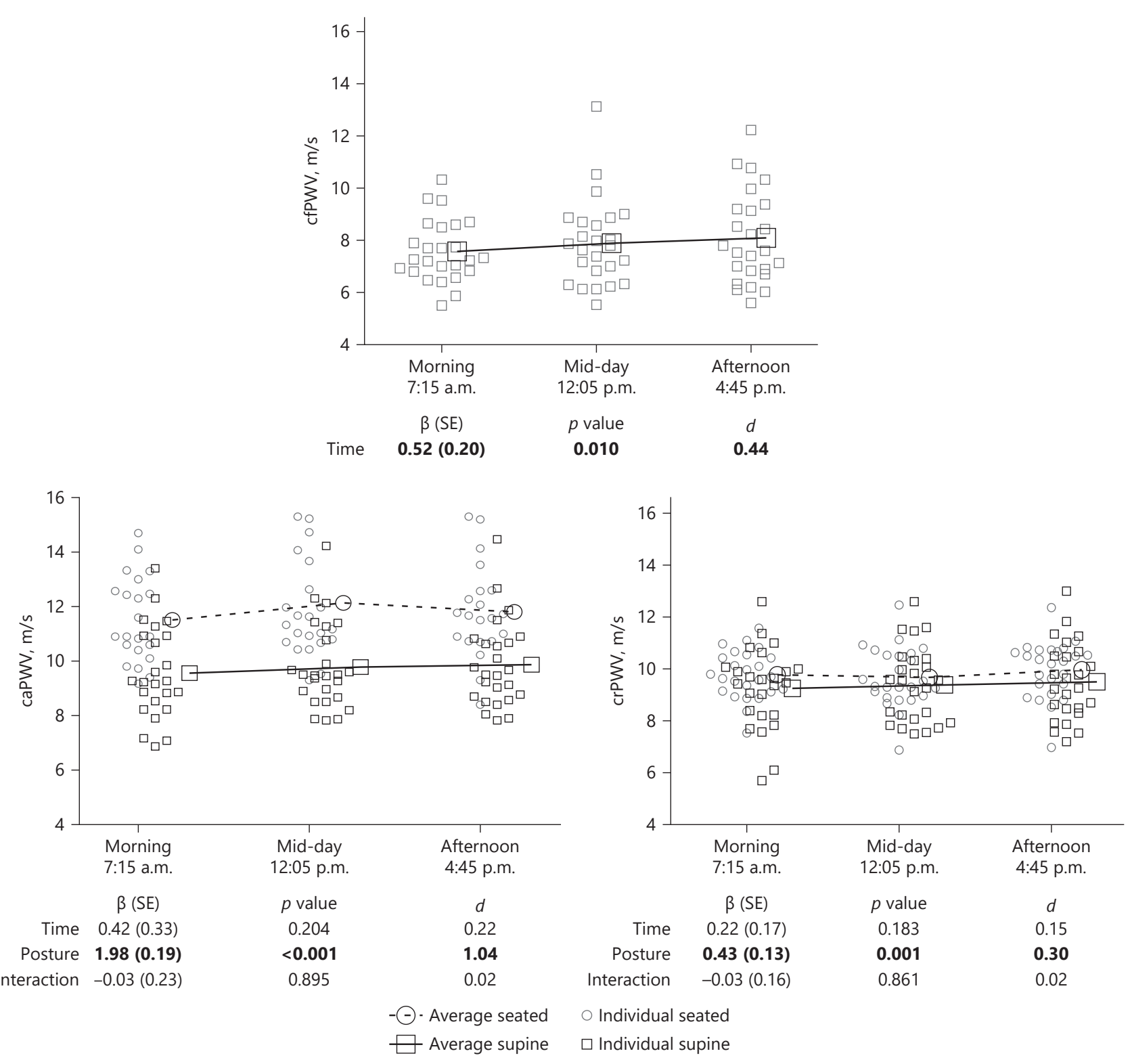

Fig. 3. PWV responses to prolonged sitting in a seated versus supine posture. GLM models examined the main effects of time, posture, and their interaction on PWV responses. $\beta$ coefficients represent change in PWV over the entire experiment (main effect of time), differences in PWV comparing sitting to standing (main

in seated SBP, DBP, and MAP and supine cfPWV and caPWV. Further, the findings suggest that the influence of posture on $\mathrm{BP}$ and $\mathrm{PWV}$ responses to prolonged sitting appears to be minimal. These results add to the evidence supporting adverse impacts of prolonged sitting on cardiovascular function. This study emphasizes current rec- effect of posture), and their interaction. GLM, generalized linear mixed; caPWV, carotid-ankle pulse wave velocity; cfPWV, carotid-femoral pulse wave velocity; crPWV, carotid-radial pulse wave velocity; $d$, Cohen's $d ; \mathrm{SE}$, standard error. Bold indicates significant effect $(p<0.05)$.

ommendations [32-34] to limit the accumulation of prolonged sitting time as it appears to acutely impair cardiovascular functions. In addition, this study provides a call for interventions that aim at sedentary behavior reductions, especially at work, to prevent prolonged sitting-related cardiovascular dysfunctions. 


\section{Effects of Prolonged Sitting during a Simulated}

Workday

As hypothesized, prolonged sitting resulted in an increase in seated BP among overweight/obese individuals with elevated BP. While some studies have found comparable adverse effects following 3,7 , or $8 \mathrm{~h}$ of prolonged sitting [9-12], others suggest no change in BP with 3 or 7 $\mathrm{h}$ of prolonged sitting $[8,35]$. Speculatively, several factors such as postural positioning when measuring $\mathrm{BP}$, allowance of restroom breaks during prolonged sitting, and participants' clinical characteristics might have contributed to these inconsistent findings. However, no clear pattern emerges across these studies that explains why some studies find a deleterious effect of prolonged sitting on BP and some studies do not. Thus, further research into the mechanisms through which prolonged sitting may increase BP could be helpful for elucidating the true effects of prolonged sitting on acute BP. Importantly, when evaluating the effects of prolonged sitting on cardiovascular outcomes, studies should take into consideration these factors that can differently affect cardiovascular outcomes and clearly report prolonged sitting measurement protocols to facilitate comparison across the existing literature.

When measured using current recommendations of a supine posture [24], a simulated workday of prolonged sitting significantly increased supine cfPWV $(0.52 \pm 0.20$ $\mathrm{m} / \mathrm{s})$ and caPWV $(0.38 \pm 0.16 \mathrm{~m} / \mathrm{s})$ over time in participants with elevated BP and overweight/obesity. We are aware of only 2 other studies that assessed the effect of $3 \mathrm{~h}$ of prolonged sitting on cfPWV $[8,13]$. In both studies, the investigators also reported significant elevation in cfPWV following prolonged sitting. Chronic exposure to prolonged sitting and resulting acute elevations in PWV, similar to the increases observed in Credeur et al. [8], Evans et al. [13], and our study, could be detrimental to cardiovascular health long term. In the current study, though cfPWV (central) and caPWV (peripheral lower extremity) increased in response to prolonged sitting when measured in a supine posture, crPWV (the peripheral upper extremity) did not significantly increase. This could reflect that the upper limbs had continued muscular action during the simulated workday (i.e., typing or writing) that could have maintained function in these vessels. This contrasts exposure to different mechanisms in the lower extremity during prolonged sitting (gravity, tortuous vessels, and lack of muscular pump) that lead to blood pooling in the legs and vascular dysfunction [36, 37].

Though limited by small sample size $(n=9$ females; $n=16$ males), our data suggest that physiological sex dif- ferences could potentially be a source of variation in cardiovascular responses to prolonged sitting. Although Vranish et al. [10] found no interaction by sex in BP or $\mathrm{HR}$ responses to $3 \mathrm{~h}$ of prolonged sitting, we detected significant interaction by sex in seated DBP and MAP responses (females $>$ males) to a simulated workday of prolonged sitting. Further, though no significant interaction by sex was observed, increases in supine PWV with prolonged sitting were more apparent $($ all $p<0.05)$ in males than in females. This finding was similar to a 3 -h prolonged sitting study by Credeur et al. [8] in 13 males and 7 females. Further investigation of sex differences in cardiovascular responses to prolonged sitting is warranted.

\section{Effects of Posture on Cardiovascular Measurements} during Prolonged Sitting Experiments

Postural positioning for cardiovascular measurements during and following prolonged sitting has gained attention as a potentially important methodological consideration $[8,38]$. Postural positioning, specifically transitioning from seated to supine to perform cardiovascular measurements, may be enough of a stimulus to obscure or attenuate some of the effects of prolonged sitting. Thus, we compared BP and PWV responses to a simulated workday of prolonged sitting while maintaining a seated posture and then repeated assessments after a transition and 10-min rest in a supine posture. Though prolonged sitting over time increased SBP, the magnitude of the effects was larger in the seated versus supine measurements for all BP measures (SBP: $d=0.43$ vs. 0.28 , DBP: $d=0.30$ vs. 0.13 , and MAP: $d=0.40$ vs. 0.18 , respectively). As described below, the greater magnitude of seated compared to supine BP responses could be due to physiological mechanisms that promote greater seated, compared to supine, cardiovascular responses to the simulated workday of prolonged sitting.

Although, central PWV (cfPWV) was not measured in the seated posture due to the lack of access to the femoral artery, peripheral PWV (crPWV and caPWV) was obtained in both postures. crPWV responses to the simulated workday did not change significantly over time when measured in either a seated or supine posture. Furthermore, caPWV had a similar magnitude of increase in both seated and supine postures, but the increase was only statistically significant during the supine measurement. It is possible that the difference in statistical significance for caPWV responses is attributable to greater variability and measurement error in the seated posture, as has been previously reported [38]. Moreover, the smaller overall increases in seated compared to supine 
PWV could potentially be due to a ceiling effect, where high values of PWV are less likely to increase further [39, 40]. Nevertheless, we are unaware of other studies comparing PWV responses to prolonged sitting measured in different postures; future studies are needed to explore the mechanisms that may explain differences in PWV responses in a seated versus supine posture.

\section{Plausible Mechanisms}

Several mechanisms, such as alpha (mainly found in vessels) and beta (mainly found in the heart) sympathetic overactivation, which can lead to increased BP, PWV, and HR $[41,42]$, have been proposed to explain the effects of prolonged sitting on cardiovascular function [43]. One theory suggests that assuming a quiet, seated posture where the trunk and lower extremity are vertically positioned on a chair and floor, respectively, results in blood pooling in the lower extremity due to gravitational force and diminished muscular pump. This leads to a decrease in venous return (preload), ventricular filling, cardiac output, and MAP [22]. To compensate, neural mechanisms, specifically alpha and beta receptors, are activated leading to decreases in venous compliance and increases in systemic vascular resistance and HR [22, 44, 45]. Over a period, these mechanisms may be overactivated which may lead to increased BP, HR, and, potentially, PWV. In this study, we observed increases in seated SBP, DBP, and MAP, which may imply increased activation of alpha sympathetic receptors. We also noted that seated HR did not significantly change, and if anything, tended to be lower at the time of cardiovascular assessments following the simulated workday of prolonged sitting. This HR response could potentially indicate that beta sympathetic receptors did not change; nevertheless, because HR is regulated by both branches of the autonomic nervous system (sympathetic and parasympathetic) which can function reciprocally or nonreciprocally [46], caution should be taken when interpreting HR response in this study. It is possible that the beta sympathetic activity has increased but is accompanied with equivalent increases in parasympathetic outflow to the heart, leading to unchanged HR. However, without more direct measurements of autonomic nervous system activity, this hypothesis cannot be confirmed from our study and warrants further investigation.

We hypothesized that these mechanisms and cardiovascular responses would be different when measured in a supine compared to seated posture. We expected that interrupting prolonged sitting by transitioning to a supine posture could interfere with these mechanisms or promote counterpart mechanisms. For example, in a supine posture, the whole body is vertically positioned on a bed or table and gravitational forces are distributed across the whole body. This is in contrast to the gravitational force during sitting that would be more strongly exerted on the lower extremity. Consequently, transitioning from a seated to supine posture for vascular assessments could promote recirculation and interfere with pooled blood in the lower extremity that is observed during prolonged sitting. In that case, the neural responses, specifically alpha and beta sympathetic activity, would be different. In fact, several studies have demonstrated greater vascular (alpha) sympathetic activity in muscles when seated compared to supine [47, 48]. While we did not test this mechanism explicitly, our findings are consistent with this hypothesis. Though no significant interactions were observed as mentioned earlier, the magnitude of the effects of prolonged sitting on SBP, DBP, and MAP was about twofold greater when measured in the seated compared to supine posture. This could be due to greater sympathetic activation when seated compared to supine as observed in other studies [47, 48].

\section{Limitations and Strengths}

There were limitations that should be considered when interpreting our findings. Our sample included both sex groups ( $16 \mathrm{M} ; 9 \mathrm{~F}$ ) and a wide range of age ( $42 \pm 12$ years old) to increase the generalizability of the findings. Yet, this may have introduced some variability, and our small sample size in this secondary analysis may also have led to a lack of statistical power.

The lack of a comparison control condition for evaluating overall responses to prolonged sitting is another limitation. In acute physical activity or other behavioral research, sitting is often the control condition. However, due to recent growing evidence showing the potential detrimental effects, prolonged sitting may now be considered as the experimental condition. Further, because this study aimed to evaluate the effects of a simulated workday of prolonged sitting, participants were only instructed to remain seated without any further restrictions on their lower limb motion or mode (i.e., not instructed to avoid fidgeting or leg crossing), which could have affected cardiovascular responses [37, 49].

The postures, by logistical limitation of needing to maintain the prolonged seated posture, could not be counterbalanced. All measurements were always completed in the same order, seated followed by the supine measurement, and this could have introduced systematic bias. During this postural transitioning (from seated to 
supine), participants took a couple of steps from the chair to the bed for assessment. This transitioning, while likely a part of many other study protocols like ours, could have interfered with the true effects of prolonged sitting on the cardiovascular outcomes beyond the simple postural change. Lastly, measurement error was more likely to exist during the seated caPWV measurement due to the small size and anatomical position of the vessel which creates more technical challenges. This was reflected in the standard errors when comparing seated versus supine caPWV.

Though we did not evaluate the postmenopausal status or control for the menstrual cycle phase for female participants as this is not typically included as a recommended practice for the measurement of $\mathrm{BP}$ and $\mathrm{PWV}$ $[24,26]$, these could have affected cardiovascular responses to the simulated workday of prolonged sitting [50-52] and are thus a limitation of our study.

The study also has several strengths that are worth mentioning. The study evaluated cardiovascular responses to a simulated workday $(>7 \mathrm{~h}$ ) of prolonged sitting which mimicked a typical workday and contrasts other studies that have utilized shorter duration $(\leq 3 \mathrm{~h})$ of prolonged sitting $[8,10,45,53]$. This study also compared cardiovascular responses to prolonged sitting when measured in seated and supine postures, informing protocol design for future prolonged sitting studies. Most of the previous studies evaluated cardiovascular responses to prolonged sitting in only 1 posture $[9,44,45$, 53]. Further, our study had direct observation of posture and activity, dietary intake was standardized, and we used the gold standard, objective assessment of cardiovascular responses.

\section{Conclusion}

In sum, seated SBP, DBP, and MAP and supine cfPWV and caPWV increased across a simulated workday of prolonged sitting. The influence of posture on these responses appears to be minimal. Over time, these increases in BP and PWV, especially cfPWV, could lead to exaggerated cardiovascular workload, reduced coronary artery perfusion, and, eventually, cardiovascular diseases [54]. Thus, our findings add to the growing body of evidence suggesting the adverse impact that acute exposure to prolonged, workplace sitting has on cardiovascular function. These findings support the development of interventions and recommendations to reduce and break prolonged sitting to mitigate its negative cardiovascular effects $[32-34,55]$.
In addition, the effects of prolonged sitting on $\mathrm{BP}$ and PWV were minimal and not statistically different across postures. Still, BP responses to prolonged sitting were nonsignificantly greater when seated, and PWV responses had greater precisions when supine; these factors could be additionally considered when designing measurement protocols in future prolonged sitting experiments.

Future research should study the long-term effects of chronic exposure to prolonged sitting on BP, PWV, and cardiovascular health. Studies should also directly investigate mechanisms, such as shear stress, venous return, and alpha and beta sympathetic outflow, in response to prolonged sitting, during both seated and supine cardiovascular measurements.

\section{Acknowledgement}

The authors would like to thank all participants for their participation.

\section{Statement of Ethics}

All participants gave their written informed consent. All the study's procedures were approved by the Institutional Review Board at the University of Pittsburgh.

\section{Conflict of Interest Statement}

A.B.A., R.J.K., M.A.J., S.J.P., L.S., and B.B.G. disclose no conflicts of interest.

\section{Funding Sources}

The parent research study providing data for this secondary analysis was supported by an investigator-initiated grant from $\mathrm{Hu}$ manscale awarded to B.B.G. and was supported by the National Institute of Health through Grant No. UL1TR000005 (University of Pittsburgh CTSI, providing research registry support).

\section{Author Contributions}

B.B.G. designed the study. B.B.G., R.J.K., M.A.J., and S.J.P. performed the experiments. A.B.A. and B.B.G. analyzed and interpreted the data and wrote the first manuscript. A.B.A., B.B.G., R.J.K., M.A.J., S.J.P., and L.S. reviewed, revised, and approved the final manuscript. 


\section{References}

1 Tremblay MS, Aubert S, Barnes JD, Saunders TJ, Carson V, Latimer-Cheung AE, et al. Sedentary Behavior Research Network (SBRN)-Terminology Consensus Project process and outcome. Int J Behav Nutr Phys Act. 2017;14(1):75.

2 Biddle SJ, Bennie JA, Bauman AE, Chau JY, Dunstan D, Owen N, et al. Too much sitting and all-cause mortality: is there a causal link? BMC Public Health. 2016;16(1):635.

3 Dunstan DW, Thorp AA, Healy GN. Prolonged sitting: is it a distinct coronary heart disease risk factor? Curr Opin Cardiol. 2011;26(5):412-9.

4 Tudor-Locke C, Leonardi C, Johnson WD, Katzmarzyk PT. Time spent in physical activity and sedentary behaviors on the working day: the American time use survey. J Occup Environ Med. 2011;53(12):1382-7.

5 Biswas A, Oh PI, Faulkner GE, Bajaj RR, Silver MA, Mitchell MS, et al. Sedentary time and its association with risk for disease incidence, mortality, and hospitalization in adults: a systematic review and meta-analysis. Ann Intern Med. 2015;162(2):123-32.

6 Barone Gibbs B, Kowalsky RJ, Perdomo SJ, Taormina JM, Balzer JR, Jakicic JM. Effect of alternating standing and sitting on blood pressure and pulse wave velocity during a simulated workday in adults with overweight/ obesity. J Hypertens. 2017;35(12):2411-8.

7 Bailey DP, Locke CD. Breaking up prolonged sitting with light-intensity walking improves postprandial glycemia, but breaking up sitting with standing does not. J Sci Med Sport. 2015;18(3):294-8.

8 Credeur DP, Miller SM, Jones R, Stoner L, Dolbow DR, Fryer SM, et al. Impact of prolonged sitting on peripheral and central vascular health. Am J Cardiol. 2019;123(2):260-6.

9 Larsen RN, Kingwell BA, Sethi P, Cerin E, Owen N, Dunstan DW. Breaking up prolonged sitting reduces resting blood pressure in overweight/obese adults. Nutr Metab Cardiovasc Dis. 2014;24(9):976-82.

10 Vranish JR, Young BE, Kaur J, Patik JC, Padilla J, Fadel PJ. Influence of sex on microvascular and macrovascular responses to prolonged sitting. Am J Physiol Heart Circ Physiol. 2017;312(4):H800-5.

11 O’Brien MW, Johns JA, Williams TD, Kimmerly DS. Sex does not influence impairments in popliteal endothelial-dependent vasodilator or vasoconstrictor responses following prolonged sitting. J Appl Physiol (1985). 2019;127(3):679-87.

12 Zeigler ZS, Mullane SL, Crespo NC, Buman MP, Gaesser GA. Effects of standing and light-intensity activity on ambulatory blood pressure. Med Sci Sports Exerc. 2016;48(2): 175-81.

13 Evans WS, Stoner L, Willey Q, Kelsch E, Credeur DP, Hanson ED. Local exercise does not prevent the aortic stiffening response to acute prolonged sitting: a randomized crossover trial. J Appl Physiol (1985). 2019;127(3):7817.

Vascular Responses to Prolonged Sitting
14 Hitosugi M, Niwa M, Takatsu A. Rheologic changes in venous blood during prolonged sitting. Thromb Res. 2000;100(5):409-12.

15 Naka KK, Tweddel AC, Doshi SN, Goodfellow J, Henderson AH. Flow-mediated changes in pulse wave velocity: a new clinical measure of endothelial function. Eur Heart J. 2006;27(3):302-9.

16 Takahashi T, Hayano J, Okada A, Saitoh T, Kamiya A. Effects of the muscle pump and body posture on cardiovascular responses during recovery from cycle exercise. Eur J Appl Physiol. 2005;94(5-6):576-83.

17 Farinatti PdTV, Nakamura FY, Polito MD. Influence of recovery posture on blood pressure and heart rate after resistance exercises in normotensive subjects. J Strength Cond Res. 2009;23(9):2487-92.

18 Fleg JL, O’Connor F, Gerstenblith G, Becker LC, Clulow J, Schulman SP, et al. Impact of age on the cardiovascular response to dynamic upright exercise in healthy men and women. J Appl Physiol (1985). 1995;78(3):890-900.

19 Smith JJ, Porth CM, Erickson M. Hemodynamic response to the upright posture. J Clin Pharmacol. 1994;34(5):375-86.

20 Pomeranz B, Macaulay RJ, Caudill MA, Kutz I, Adam D, Gordon D, et al. Assessment of autonomic function in humans by heart rate spectral analysis. Am J Physiol. 1985;248(1 Pt 2):H151-3.

21 Harper CM, Lyles YM. Physiology and complications of bed rest. J Am Geriatr Soc. 1988; 36(11):1047-54.

22 Shvartz E, Gaume JG, White RT, Reibold RC. Hemodynamic responses during prolonged sitting. J Appl Physiol Respir Environ Exerc Physiol. 1983;54(6):1673-80.

23 Cohen J, Pignanelli C, Burr J. The effect of body position on measures of arterial stiffness in humans. J Vasc Res. 2020;57(3):143-51.

24 Van Bortel LM, Duprez D, Starmans-Kool MJ, Safar ME, Giannattasio C, Cockcroft J, et al. Clinical applications of arterial stiffness, Task Force III: recommendations for user procedures. Am J Hypertens. 2002;15(5):445-52.

25 Mahe G, Comets E, Nouni A, Paillard F, Dourmap C, Le Faucheur A, et al. A minimal resting time of $25 \mathrm{~min}$ is needed before measuring stabilized blood pressure in subjects addressed for vascular investigations. Sci Rep. 2017;7(1):12893-8.

26 Whelton P, Carey R, Aronow W, Casey D Jr, Collins K, Dennison H, et al. 2017 ACC/ AHA/AAPA/ABC/ACPM/AGS/APhA/ ASH/ASPC/NMA/PCNA guideline for the prevention, detection, evaluation, and management of high blood pressure in adults: a report of the American College of Cardiology/American Heart Association Task Force on clinical practice guidelines. Hypertension. 2018;71(6):1269-324.

27 Chobanian AV, Bakris GL, Black HR, Cushman WC, Green LA, Izzo JL Jr, et al. Seventh report of the joint national committee on pre- vention, detection, evaluation, and treatment of high blood pressure. Hypertension. 2003; 42(6):1206-52.

28 Harris JA, Benedict FG. A biometric study of human basal metabolism. Proc Natl Acad Sci U S A. 1918;4(12):370.

29 Coleman A, Freeman P, Steel S, Shennan A. Validation of the Omron 705IT (HEM759-E) oscillometric blood pressure monitoring device according to the British Hypertension Society protocol. Blood Press Monit. 2006;11(1):27-32.

30 Iglesias PB, Mayoral ES, Lapetra JP, Iborra MO, Villalba FA, Cayuela AD. Validation of two systems of self-measurement of blood pressure, the OMRON HEM-705 CP and OMRON M1 (HEM 422C2-E) models. Aten Primaria. 2002;30(1):22-8.

31 Kowalsky RJ, Stoner L, Perdomo SJ, Taormina JM, Jones MA, Credeur DP, et al. Validity and reliability of peripheral pulse wave velocity measures in a seated posture. Hypertension Res. 2020;43:845-7.

32 Piercy KL, Troiano RP, Ballard RM, Carlson SA, Fulton JE, Galuska DA, et al. The physical activity guidelines for Americans. JAMA. 2018;320(19):2020-8.

33 Buckley JP, Hedge A, Yates T, Copeland RJ, Loosemore M, Hamer M, et al. The sedentary office: an expert statement on the growing case for change towards better health and productivity. Br J Sports Med. 2015;49(21):1357-62.

34 Australian Government Department of Health. 2014. Australia's physical activity and sedentary behaviour guidelines.

35 Wennberg $\mathrm{P}$, Boraxbekk CJ, Wheeler $\mathrm{M}$, Howard B, Dempsey PC, Lambert G, et al. Acute effects of breaking up prolonged sitting on fatigue and cognition: a pilot study. BMJ Open. 2016;6(2):e009630.

36 Restaino RM, Holwerda SW, Credeur DP, Fadel PJ, Padilla J. Impact of prolonged sitting on lower and upper limb micro- and macrovascular dilator function. Exp Physiol. 2015; 100(7):829-38.

37 Morishima T, Restaino RM, Walsh LK, Kanaley JA, Fadel PJ, Padilla J. Prolonged sitting-induced leg endothelial dysfunction is prevented by fidgeting. Am J Physiol Heart Circ Physiol. 2016;311(1):H177-82.

38 Stone K, Fryer S, Kelsch E, Burnet K, Zieff G, Faulkner J, et al. Validity and reliability of lower-limb pulse-wave velocity assessments using an oscillometric technique. Exp Physiol. 2019;104(5):765-74.

39 Moyen NE, Ganio MS, Burchfield JM, Tucker MA, Gonzalez MA, Dougherty EK, et al. Effect of passive heat stress on arterial stiffness in smokers versus non-smokers. Int J Biometeorol. 2016;60(4):499-506.

40 Kim T, Lee CS, Lee SD, Kang SH, Han JW, Malhotra A, et al. Impacts of comorbidities on the association between arterial stiffness and obstructive sleep apnea in the elderly. Respiration. 2015;89(4):304-11. 
41 Pang CC. Autonomic control of the venous system in health and disease: effects of drugs. Pharmacol Ther. 2001;90(2-3):179-230.

42 Bruno RM, Ghiadoni L, Seravalle G, Dell'Oro R, Taddei S, Grassi G. Sympathetic regulation of vascular function in health and disease. Front Physiol. 2012;3:284.

43 Dempsey PC, Larsen RN, Dunstan DW, Owen N, Kingwell BA. Sitting less and moving more: implications for hypertension. Hypertension. 2018;72(5):1037-46.

44 Dempsey PC, Sacre JW, Larsen RN, Straznicky NE, Sethi P, Cohen ND, et al. Interrupting prolonged sitting with brief bouts of light walking or simple resistance activities reduces resting blood pressure and plasma noradrenaline in type 2 diabetes. J Hypertens. 2016; 34(12):2376-82.

45 McManus AM, Ainslie PN, Green DJ, Simair RG, Smith K, Lewis N. Impact of prolonged sitting on vascular function in young girls. Exp Physiol. 2015;100(11):1379-87.
46 Shaffer F, Ginsberg JP. An overview of heart rate variability metrics and norms. Front Public Health. 2017;5:258.

47 Ray CA, Rea RF, Clary MP, Mark AL. Muscle sympathetic nerve responses to dynamic onelegged exercise: effect of body posture. Am J Physiol. 1993;264(1 Pt 2):H1-7.

48 Burke D, Sundlöf G, Wallin G. Postural effects on muscle nerve sympathetic activity in man. J Physiol. 1977;272(2):399-414.

49 Tremblay JC, Stimpson TV, Murray KM, Pyke KE. Sitting cross-legged for 30 min alters lower limb shear stress pattern but not flowmediated dilation or arterial stiffness. Appl Physiol Nutr Metab. 2019;44(2):221-4.

50 Hayashi K, Miyachi M, Seno N, Takahashi K, Yamazaki K, Sugawara J, et al. Variations in carotid arterial compliance during the menstrual cycle in young women. Exp Physiol. 2006;91(2):465-72.

51 Williams MR, Westerman RA, Kingwell BA, Paige J, Blombery PA, Sudhir K, et al. Varia- tions in endothelial function and arterial compliance during the menstrual cycle. Clin Endocrinol Metab. 2001;86(11):538995.

52 Serviente C, Witkowski S. Follicle-stimulating hormone, but not cardiorespiratory fitness, is associated with flow-mediated dilation with advancing menopausal stage. Menopause. 2019;26(5):531-9.

53 Thosar SS, Bielko SL, Mather KJ, Johnston JD, Wallace JP. Effect of prolonged sitting and breaks in sitting time on endothelial function. Med Sci Sports Exerc. 2015;47(4):843-9.

54 DeLoach SS, Townsend RR. Vascular stiffness: its measurement and significance for epidemiologic and outcome studies. Clin J Am Soc Nephrol. 2008;3(1):184-92.

55 Bhammar DM, Sawyer BJ, Tucker WJ, Gaesser GA. Breaks in sitting time: effects on continuously monitored glucose and blood pressure. Med Sci Sports Exerc. 2017;49(10): 2119-30. 